\title{
Optical Heating and Temperature Determination of Core- Shell Gold Nanoparticles and Single-Walled Carbon Nanotube Microparticles
}

\author{
Alexey Yashchenok, * Admir Masic, * Dmitry Gorin, Olga Inozemtseva, \\ Bong Sup Shim, Nicholas Kotov, Andre Skirtach, and Helmuth Möhwald
}

\begin{abstract}
The real-time temperature measurement of nanostructured materials is particularly attractive in view of increasing needs of local temperature probing with high sensitivity and resolution in nanoelectronics, integrated photonics, and biomedicine. Light-induced heating and Raman scattering of single-walled carbon nanotubes with adsorbed gold nanoparticles decorating silica microparticles are reported, by both green and near IR lasers. The plasmonic shell is used as nanoheater, while the singlewalled carbon nanotubes are Raman active and serve as a thermometer. Stokes and Anti-Stokes Raman spectra of single-walled carbon nanotubes serve to estimate the effective light-induced temperature rise on the metal nanoparticles. The temperature rise is constant with time, indicating stability of the adsorption density. The effective temperatures derived from Stokes and Anti-Stokes intensities are correlated with those measured in a heating stage. The resolution of the thermal experiments in our study was found to be 5-40 K.
\end{abstract}

Dr. A. Yashchenok, Prof. H. Möhwald

Max Planck Institute of Colloids and Interfaces Department of Interfaces

14424, Potsdam, Germany

E-mail: alexey.yashchenok@mpikg.mpg.de

Dr. A. Masic

Max-Planck Institute of Colloids and Interfaces

Department of Biomaterials

14424, Potsdam, Germany

E-mail: masic@mpikg.mpg.de

Dr. A. Yashchenok, Prof. D. Gorin, Dr. O. Inozemtseva

Saratov State University

Faculty of Nano- and Biomedical Technologies

Saratov 410012, Russia

B. S. Shim, N. Kotov

University of Michigan

Department of Chemical Engineering

Department of Materials Science

Department of Biomedical Engineering

Biointerface Institute

Ann Arbor, MI 48109, USA

Prof. A. Skirtach

Ghent University

Department of Molecular Biotechnology and Nano-Bio (NB)-Photonics

9000, Ghent, Belgium

DOI: $10.1002 /$ smll.201401697

\section{Introduction}

Heat generation in plasmonic structures by light irradiation represents an upcoming field of research termed thermoplasmonics. $^{[1]}$ Heating plasmonic nanomaterials has been realized in a number of applications including photothermal cancer therapy, ${ }^{[2]}$ remote release of molecules from polymeric and natural carriers, ${ }^{[3]}$ bioimaging, ${ }^{[4]}$ patterning, ${ }^{[5]}$ cell manipulation, ${ }^{[6]}$ optoporation ${ }^{[7]}$ and optofluidics. $^{[8]}$ From both fundamental point of view and further applications in such areas as micro- and nanoelectronics, integrated photonics and biomedicine the determination of the local temperature increase in nanostructured systems is an indispensable challenge. ${ }^{[9]}$ In the last two decades a set of approaches have been demonstrated to measure the local temperature rise in plasmonic nanostructures ranging from tip-based measurements to a number of optical assessments on the basis of simultaneous heating and temperature measurement. ${ }^{[10]}$ In spite of recent achievements in the nanothermometry further improvements toward enhanced sensitivity and resolution are required. Optics based methods for determination of the local temperature rise in nanostructured materials are of particular interest, since they can be less invasive, in particularly for biomedicine, more sensitive 
and open capabilities of three-dimensional temperature measurements.

A method for measuring the temperature and quantitative investigation of the influence of absorption, size, and surface density of gold nanoparticles within polyelectrolyte microcapsules has been developed recently. ${ }^{[11]}$ Takami et al. applied the black body radiation from irradiated nanoparticles to evaluate the temperature through the Stefan-Boltzmann law, assuming that the emission is independent of the observed wavelength, but this method requires very high temperature increases and is not very sensitive. ${ }^{[12]}$ A number of systems integrating heaters and thermometers have been recently reported for the local temperature measurement with high thermal resolution. Debasu and co-workers applied $(\mathrm{Gd}, \mathrm{Yb}, \mathrm{Er})_{2} \mathrm{O}_{3}$ luminescence nanorods as thermometers, while gold nanoparticles attached to nanorod surfaces were used as heaters. ${ }^{[13]}$ They showed the temperature assessment with high resolution of $0.3-2.0 \mathrm{~K}$ and of $4-13 \mathrm{~K}$ from the Boltzmann distribution and from Planck's law, respectively. The potential of using this luminescence nanothermometer at physiological conditions in the biologically friendly spectral window was also demonstrated. The local temperature of single $40 \mathrm{~nm}$ gold nanoparticles and lithographically prepared nanodots and nanowires was determined by a photoluminescent thin film of $\mathrm{Er}^{3+}$ doped $\mathrm{Al}_{0.94} \mathrm{Ga0} ._{06} \mathrm{~N}$ excited with a continuous wave laser. ${ }^{[14]}$ This approach is based on a temperature image of the optically excited gold nanostructures from the relative peak intensities from $\mathrm{Er}_{3+}$ photoluminescence. In the work of Rocha and co-authors neodymium-doped $\mathrm{LaF}_{3}$ core/shell nanoparticles were offered as promising luminescence nanothermometers for subtissue thermal probing. They applied gold nanorods as nanoheaters while the temperature was measured by the $\mathrm{Nd}^{3+}: \mathrm{LaF}_{3}$ nanoparticles. ${ }^{[15]}$

Raman thermal microspectrometry is another tool that has been used to measure temperature rise in microstructures with integrated lasers with high thermal and spatial resolution. ${ }^{[16]}$ The Raman thermal approach offers also the temperature assessment in nanoscale materials by measuring Stokes and Anti-Stokes Raman intensities. Recently this technique has been applied for the temperature rise in carbon materials such as single-walled carbon nanotubes, active carbon and graphite upon illumination by laser light. ${ }^{[17]}$ The electron-phonon scattering as a function of temperature of Joule-heated photons on graphene with high energy electrons has been studied with a spatial resolution of $400 \mathrm{~nm}^{[18]}$ Brown and co-workers have found that semiconductor or metallic single-walled carbon nanotubes can be excited selectively with a certain laser energy. ${ }^{[19]}$ In situ measurements of Raman spectra and the temperature were accomplished for single-walled carbon nanotubes, and reversible structural changes have been shown by micro-Raman spectrometry. ${ }^{[20]}$ Non-destructive manner and real-time signal acquisition are advantages of micro-Raman spectroscopy for assessment of the local temperature rise in nanostructured materials.

In this work we constructed a microprobe made of silica microparticles surrounded by a thin nanostructured shell. A net of single-walled carbon nanotubes (SWCNT) around silica microparticles was decorated with gold nanoparticles by the layer-by-layer technique. Here gold nanoparticles serve as nanoheaters, while single-walled carbon nanotubes are used as thermometers. Gold nanoparticles are excited either at $532 \mathrm{~nm}$ or at $785 \mathrm{~nm}$ with a continuous wave laser to induce a temperature rise in the nanoscale shell. The temperature is assessed with the same laser through in situ measurement of the Anti-Stokes and the Stokes Raman spectra. Laser light induced heating of gold nanoparticles shifts the G-phonon band of SWCNT due to the proximity of gold nanoparticles and carbon nanotubes. The derived effective temperature from measurements of Stokes and Anti-Stokes intensities is correlated to that of obtained by electric heating. The resolutions of thermal Raman measurements obtained in this study are of 5-40 K.

\section{Results and Discussion}

To construct the temperature sensor single-walled carbon nanotubes decorated with gold nanoparticles around silica microparticles were deposited by the layer-by-layer method. Our characterization of the engineered microstructures by TEM revealed that electroless plating completed the shell coating with tiny intersections (Figure 1b). This structure could not be achieved by the adsorption of gold nanoparticles (Figure 1a). We believe that such junctions are similar to those of a hot spot which usually refers to gold dimers or aggregated gold nanoparticles. ${ }^{[21]}$ The ability of these microparticles to generate high Raman scattering, that is surface
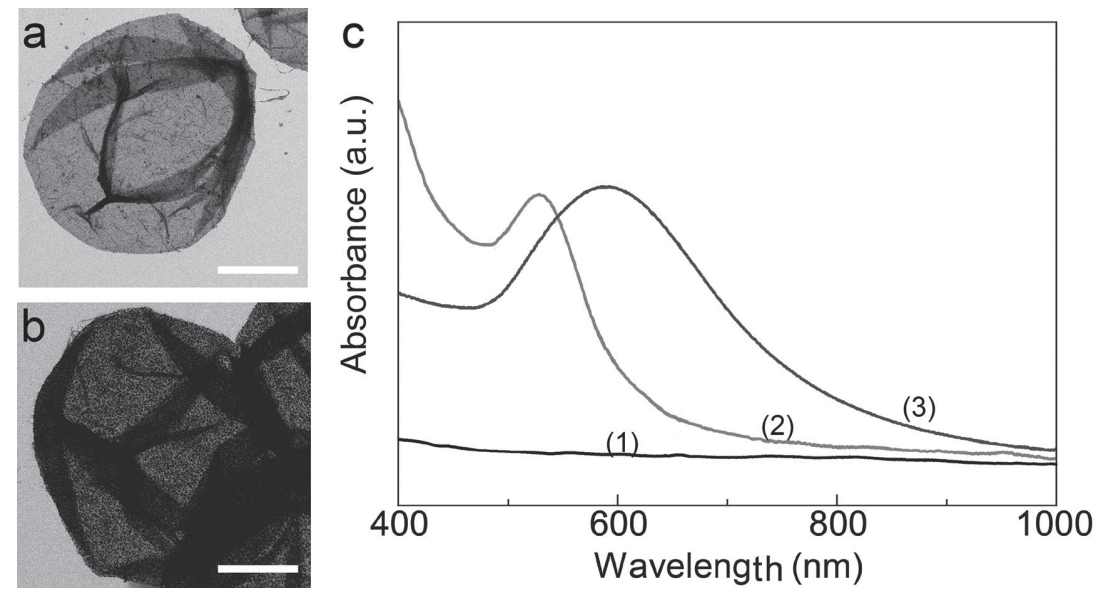

Figure 1. TEM images of microcapsules containing several layers of polyelectrolytes terminated with single-walled carbon nanotubes subsequently decorated with gold nanoparticles (PSTA sample) (a); and those after the electroless plating (PSTAS) (b). Silica microparticles were used as a template for microcapsules fabrication. UV-vis absorption spectra of the polyelectrolyte films with single-walled carbon nanotubes (line 1), gold nanoparticle suspension (line 2); single-walled carbon nanotubes decorated with gold nanoparticles by electroless plating (line 3) (3). The concentrations for deposition were used similar to those adsorbed on colloidal probes. The scale bars on the TEM images are $2 \mu \mathrm{m}$. 

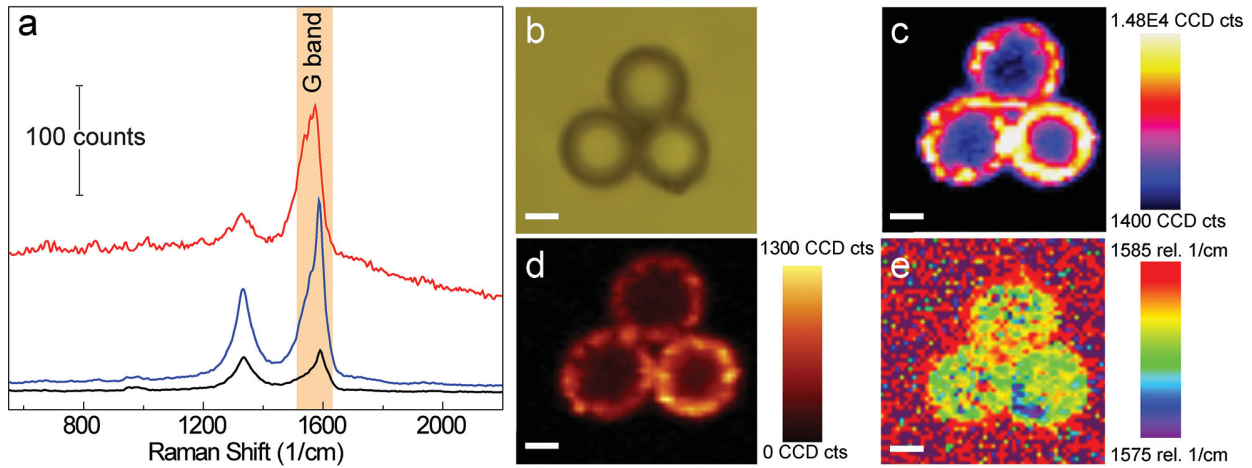

Figure 2. Raman spectra of shells surrounding the silica microparticles: PST (black curve); PSTA (blue curve); PSTAS (red curve). The spectra were acquired with $532 \mathrm{~nm}$ wavelength of the laser operating at $4 \mathrm{~mW}\left(12.7 \cdot 10^{4} \mathrm{~W} \cdot \mathrm{cm}^{-2}\right)$ through $100 \mathrm{x}$ objectives, integration time $5 \mathrm{sec}($ left-hand side). Optical image (b) and Raman image (d) of beads after chemical growth and analysis of the Raman image using the intensity of G-band $1400-1660 \mathrm{~cm}^{-1}$ (c) and shift of the $\mathrm{G}$ peak (e). The scale bars on all images correspond to $2 \mu \mathrm{m}$.

enhanced Raman scattering (SERS), has been shown in our previous study. ${ }^{[22]}$

Raman spectra of these microparticles were measured acquiring Stokes and Anti-Stokes intensities. Figure 2 shows Raman spectra of three types of core-shell silica microparticles. The G-band shift of single-walled carbon nanotubes to lower frequency at a power density of $12.7 \cdot 10^{4} \mathrm{~W} \cdot \mathrm{cm}^{-2}$ for the particles after electroless plating is attributed to heating of carbon nanotubes. A shift of about $16 \mathrm{~cm}^{-1}$ was observed for those microparticles, while other samples did not show any changes of the $G$ peak position in this power range (Table 1). One observes also a broadening of the G-band for the samples after electroless plating at $4 \mathrm{~mW}\left(12.7 \cdot 10^{4} \mathrm{~W} \cdot \mathrm{cm}^{-2}\right)$ of laser power. It is suggested that the broadening is due to a planar distortion of the G-band. This was also observed at lower intensities, however it is reversible only at a power density up to $6.4 \cdot 10^{4} \mathrm{~W} \cdot \mathrm{cm}^{-2}$ (Figure S1). As was mentioned above the electroless plating increases the amount of gold nanoparticles on the surface of single-walled carbon nanotubes, and at the same time the distance between gold nanoparticles is reduced (Figure 1b). Obviously the heating of carbon nanotubes under laser excitation would be more pronounced for gold nanoshells, rather than for separated gold nanoparticles. Raman imaging shows that the signal of the $G$ peak is uniformly distributed across the particle surface indicating a regular distribution of nanotubes around the silica beads (Figures 2c and 2d). Mapping of the Raman intensity in the range of $1400-1660 \mathrm{~cm}^{-1}$ demonstrates the red shift of the G-band (Figure 2e).

In order to estimate the temperature induced by the gold nanoshells upon laser illumination the Stokes and Anti-Stokes Raman spectra were acquired. The Stokes and Anti-Stokes intensities of the $G$ peak as a function of laser power are shown in Figure 3. Knowing the intensity ratio of the Stokes and Anti-Stokes lines it is possible to calculate the effective temperature (G-phonons occupation) by using the following equation:

$$
\frac{I_{A S}}{I_{S}}=\left(\frac{v_{0}+v_{G}}{v_{0}-v_{G}}\right)^{4} \exp \left(-\frac{\hbar v_{G}}{k_{B} T_{G}}\right),
$$

where $v_{0}$ is the excitation frequency, $v_{G}$ the G-phonon frequency, $\hbar$ and $k_{B}$ the Planck and Boltzmann constants, respectively. In order to estimate the effective temperature the Stokes and Anti-Stokes spectra were fitted with

Table 1. The centre of the $G$-band of the Stokes $\left(G_{S}\right)$ and Anti-Stokes $\left(G_{A S}\right)$ lines of the core-shell structures of Sample PST, Sample PSTA, Sample PSTAS as a function of the laser power density. The effective temperature is estimated through the ratio of the Anti-Stokes and Stokes intensities.

\begin{tabular}{|c|c|c|c|c|c|c|c|c|c|}
\hline \multirow{2}{*}{$\begin{array}{l}\text { Sample } \\
\text { Power density } \\
{\left[10^{4} \mathrm{~W} \cdot \mathrm{cm}^{-2}\right]} \\
\end{array}$} & \multicolumn{3}{|c|}{ Sample PST } & \multicolumn{3}{|c|}{ Sample PSTA } & \multicolumn{3}{|c|}{ Sample PSTAS } \\
\hline & $\mathrm{G}_{\mathrm{S}}$ & $\mathrm{G}_{\mathrm{AS}}$ & $\mathrm{T}[\mathrm{K}]$ & $\mathrm{G}_{\mathrm{S}}$ & $\mathrm{G}_{\mathrm{AS}}$ & $\mathrm{T}[\mathrm{K}]$ & $\mathrm{G}_{\mathrm{S}}$ & $\mathrm{G}_{\mathrm{AS}}$ & $\mathrm{T}[\mathrm{K}]$ \\
\hline 1.6 & 1592 & - & & 1592 & - & & 1592 & - & 298 \\
\hline 3.2 & 1592 & - & & 1592 & - & & 1587 & - & 298 \\
\hline 6.4 & 1592 & - & & 1592 & - & & 1583 & 1582 & 346 \\
\hline 9.6 & 1592 & - & & 1591 & - & & 1579 & 1577 & 462 \\
\hline 12.7 & 1592 & - & & 1590 & - & & 1575 & 1571 & 561 \\
\hline 15.9 & 1592 & - & & 1589 & - & & & & \\
\hline 31.8 & 1592 & - & & 1587 & 1586 & 348 & & & \\
\hline 47.8 & 1589 & - & & 1580 & 1580 & 487 & & & \\
\hline 63.7 & 1588 & 1587 & 365 & & & & & & \\
\hline 79.6 & 1587 & 1586 & 372 & & & & & & \\
\hline
\end{tabular}



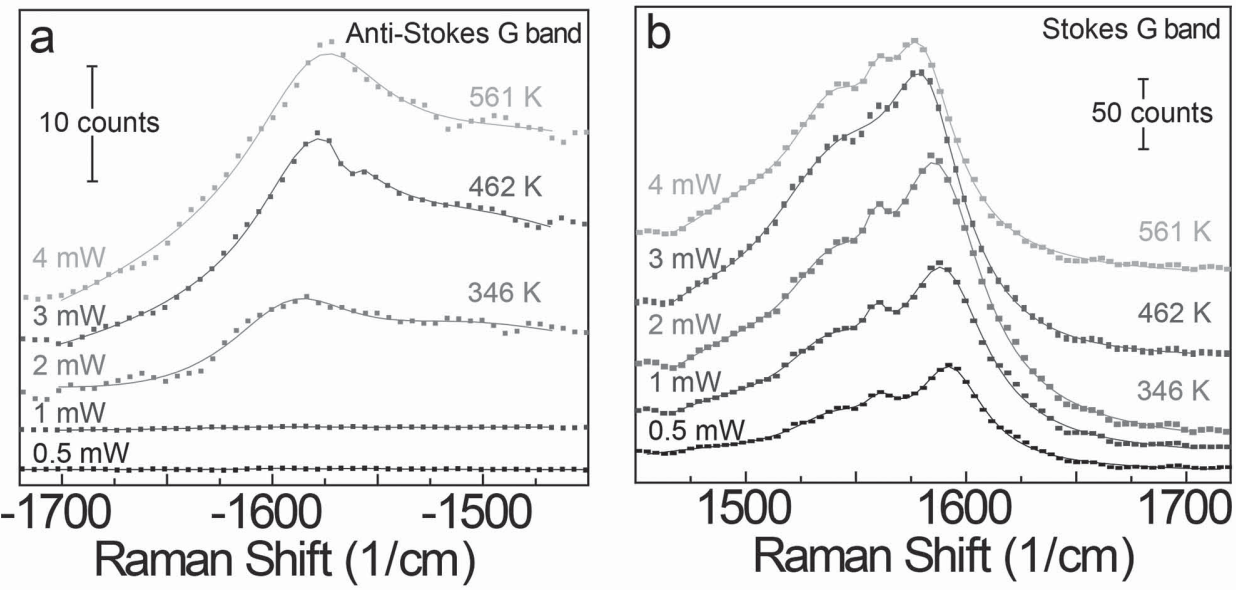

Figure 3. Anti-Stokes and Stokes Raman spectra of the PSTAS silica beads at different powers of the green laser line. The spectra were acquired with $532 \mathrm{~nm}$ wavelength of the laser operating at $4 \mathrm{~mW}\left(12.7 \cdot 10^{4} \mathrm{~W} \cdot \mathrm{cm}^{-2}\right)$ through $100 \times$ objectives, integration time $5 \mathrm{sec}$. The evaluated effective temperature is indicated above the spectra taken at a certain laser power.

Lorentzians. The temperature is evaluated through calculation of the Stokes and Anti-Stokes intensity ratio according to the above equation for the photon energy equal to $2.33 \mathrm{eV}$. The results of the temperature determination are included above each curve in Figure 3. One can see that an Anti-Stokes line of gold nanoshells on carbon nanotubes is observed for the laser power density starting from $6.4 \cdot 10^{4} \mathrm{~W} \cdot \mathrm{cm}^{-2}$, which corresponds to $346 \mathrm{~K}$. Further increase of the laser power to $4 \mathrm{~mW}\left(12.7 \cdot 10^{4} \mathrm{~W} \cdot \mathrm{cm}^{-2}\right)$ raises the temperature to $561 \mathrm{~K}$. On the other hand, the absence of Anti-Stokes lines for beads with only carbon nanotubes indicates that laser light does not induce local heating of SWCNT at lower laser powers. Substantial changes of the Anti-Stokes intensity of the G-band for those microparticles was observed only at a power density of $63.7 \cdot 10^{4} \mathrm{~W} \cdot \mathrm{cm}^{-2}$. The effective temperature for the microprobe containing only carbon nanotubes at this power level is about $343 \mathrm{~K}$. This value corresponds to an effective temperature, which was estimated for gold nanoshells at a power density of $6.4 \cdot 10^{4} \mathrm{~W} \cdot \mathrm{cm}^{-2}$. The derived temperature is correlated with the data obtained for single-walled carbon nanotubes illuminated with the $514 \mathrm{~nm}$ laser in the work of $\mathrm{Li}^{[17]}$ The temperature coefficient of the planar stretching of the G-band $\left(\mathrm{E}_{2 \mathrm{~g}}\right)$ was derived to be $-4.5 \cdot 10^{-2} \mathrm{~cm}^{-1} \cdot \mathrm{K}^{-1}\left(\mathrm{R}^{2}=0.97\right)$. Interestingly that probe can be heated by near infrared light $(785 \mathrm{~nm})$ starting from $8 \mathrm{~W} / \mathrm{cm}^{2}$ of incidence power. Figure 4 shows the temperature calculated from Stokes and AntiStokes intensities as a function of laser power density. As seen from Figure 4a, the temperature at the microparticles containing gold nanoshells increases with the increase of laser power density. When the laser power density is decreasing back a difference in the temperature has been observed. Interestingly the second run (increasing/decreasing laser power density) transferred the temperature into the same range as was obtained at the first cooling run (Figure 4b). The next heating/cooling runs did not show significant hysteresis of the temperature for the same probe. For statistical purposes, we repeated the same experiments for at least ten microparticles and the similar behavior have been found. Even the estimated temperature difference between first heating and cooling runs was very similar for all microprobes. This fact could be due to defects and disorder (network like structure) in the structure of single-walled carbon nanotubes. ${ }^{[17]}$ Another explanation of the hysteresis in the first run could be explained by a change of the localized surface plasmon resonance (LSPR) of gold nanoparticles with increasing temperature. ${ }^{[23]}$ The temperature rise can induce the bleaching of the LSPR because of the broadening of the spectrum. This is due to an increase of electronphonon scattering at higher temperature. ${ }^{[24]} \mathrm{We}$ also assume that 4-(dimethylamino)pyridine capped gold nanoparticles and polystyrene sulfonate stabilized single walled carbon
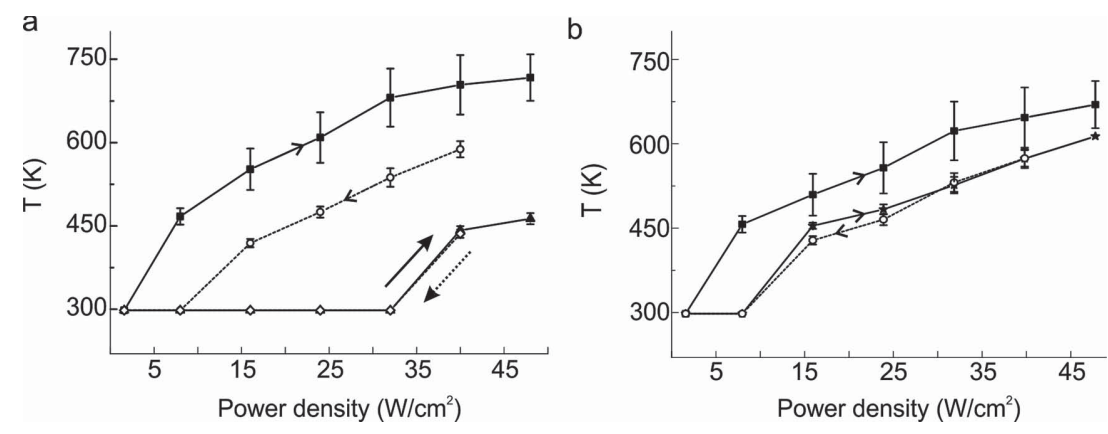

Figure 4. (a) Temperatures derived from measuring Stokes and Anti-Stokes intensities of the microprobe "single-walled carbon nanotubes decorated with gold nanoparticles" after electroless plating (filled squares and open circles), and those without plasmonic nanoshell (filled triangles and open rhombohedra) as a function of laser power density. (b) Temperature derived from Raman measurements of the microprobe single-walled carbon nanotubes decorated with plasmonic nanoshell at first (filled squares and open circles) and second runs (filled stars and open circles) respectively. Arrows indicate heating and cooling runs. The Stokes and Anti-Stokes spectra were acquired with $785 \mathrm{~nm}$ wavelength of the laser operating at $0.1 \mathrm{~mW}\left(0.3 \cdot 10^{4} \mathrm{~W} \cdot \mathrm{cm}^{-2}\right)$ through $100 \times$ objectives, integration time $0.5 \mathrm{sec}$. 
nanotubes can induce hysteresis due to thermal isolation of gold nanoparticles from the surrounding molecules. Laser heating of gold nanoparticles can remove and/or photodecompose 4-(dimethylamino)pyridine (meting point 110-113 ${ }^{\circ} \mathrm{C}$ ) and polystyrene sulfonate $\left(\mathrm{Tg}=152{ }^{\circ} \mathrm{C}\right.$ ) capped molecules. ${ }^{[25]}$ The decomposition of capped molecules can reduce the refractive index from $\sim 1.39$ (polysterene sulfonate) to 1.0 (air). As result, these changes in the refractive index can significantly reduce the optical absorption of gold nanoparticles. ${ }^{[26]}$ Also laser induced separation of gold nanoparticles from capped molecules can lower the hysteresis due to removal of thermal insulation caused by stabilizer molecules.

The temperature at the microparticles with only singlewalled carbon nanotubes is unchanged up to $32 \mathrm{~W} \cdot \mathrm{cm}^{-2}$. Above this power density the temperature is increasing to $463 \mathrm{~K}$ at $48 \mathrm{~W} \cdot \mathrm{cm}^{-2}$. However, this temperature is lower than that at the same power density for the probe with plasmonic shell. This observation indicates that heating induced by laser light is mainly generate by gold nanoshells in selected power densities (up to $32 \mathrm{~W} \cdot \mathrm{cm}^{-2}$ ) rather than by singlewalled carbon nanotubes. Time dependent temperature measurements for two lasers with 532 and 785 nm excitation, respectively, are presented in Figure 5. These data indicate that the temperature at different power density remains stable with time. Yet, the temperature obtained at $532 \mathrm{~nm}$ can be achieved with a similar magnitude at $785 \mathrm{~nm}$, but at higher laser energies due to the lower absorption coefficient. Stability of the temperature in the entire time frame would include that the adsorption is also stable and does not change with time.

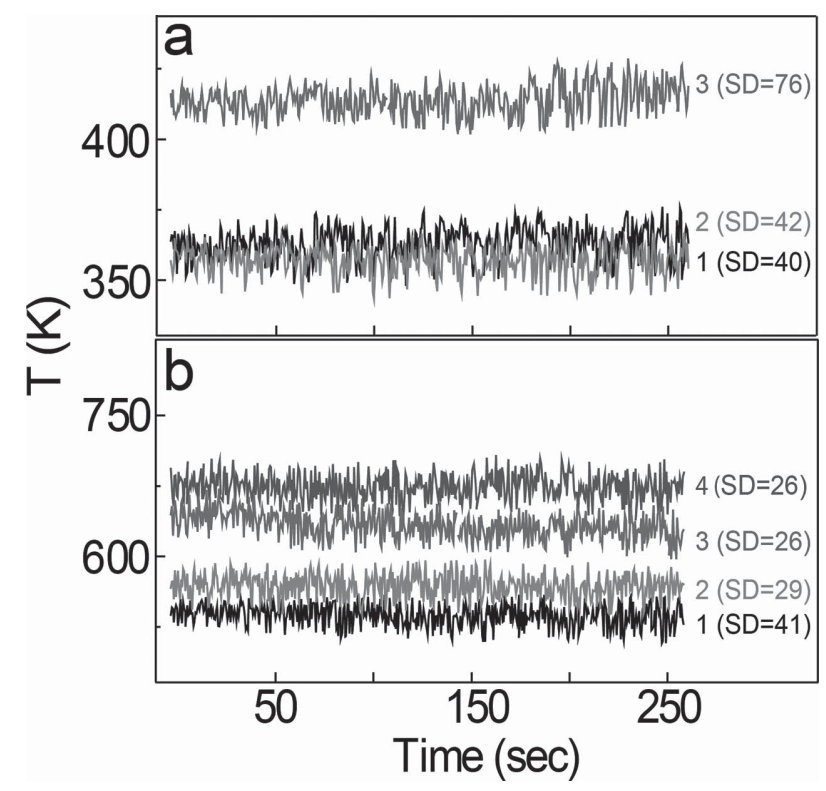

Figure 5. Time dependent temperature of the PSTAS silica beads upon illumination with a laser at $532 \mathrm{~nm}$ (a) and $785 \mathrm{~nm}$ (b). Each curve refers to a mean effective temperature calculated in accordance with Equation (1) (see in the text). The curves 1, 2, and 3 in (a) are taken at power density of $3.2 \cdot 10^{4} \mathrm{~W} \cdot \mathrm{cm}^{-2}, 6.4 \cdot 10^{4} \mathrm{~W} \cdot \mathrm{cm}^{-2}, 9.6 \cdot 10^{4} \mathrm{~W} \cdot \mathrm{cm}^{-2}$ respectively. The curves $1,2,3$ and 4 in (b) were obtained at a power density $16 \cdot 10^{4} \mathrm{~W} \cdot \mathrm{cm}^{-2}, 2410^{4} \mathrm{~W} \cdot \mathrm{cm}^{-2}, 32 \cdot 10^{4} \mathrm{~W} \cdot \mathrm{cm}^{-2}, 40 \cdot 10^{4} \mathrm{~W} \cdot \mathrm{cm}^{-2}$. The standard deviation for each power density is shown in the brackets. Each curve was compressed for clarity.
As has been shown earlier, the temperature change is related to the heating rate per unit volume per unit time, the thermal conductivity of the surrounding medium, the size of absorbing centers, their specific heat capacity and thermal conductivity. ${ }^{[27]}$ The heating rate is proportional to the incident power density, absorption of heating centers and their concentration. ${ }^{[27]}$ The extinction coefficient of gold nanoparticles at the maximum adsorption is higher than that for carbon nanotubes (Figure 1c). The specific heat capacity and thermal conductivity for gold $\left(129 \mathrm{~J} \cdot \mathrm{kg}^{-1} \cdot \mathrm{K}^{-1}, 318 \mathrm{~W} \cdot \mathrm{mK}^{-1[28 \mathrm{a}]}\right.$ is lower than for carbon nanotubes $\left(600 \mathrm{~J} \mathrm{~kg}^{-1} \cdot \mathrm{K}^{-1}\right.$,[28b] $950 \mathrm{~W} \cdot \mathrm{mK}^{-1}$ along the axis of the nanotube ${ }^{[28 \mathrm{c}]}$. Taking into account the extension coefficient, the heat capacity and the thermal coefficient of gold nanoparticles and single-walled carbon nanotubes one can conclude that the heating rate for gold nanoparticles is higher than for carbon nanotubes. As consequence gold nanoparticles are heated faster at selected laser powers than SWCNT. In our experiments the laser spot was found to be about $1 \mu \mathrm{m}$ in diameter, while the microparticle diameter was $4.8 \mu \mathrm{m}$. In this case heat is generated only by those gold nanoparticles which are in the laser spot and then due to the proximity between gold nanoparticles and single-walled carbon nanotubes is propagated to carbon nanotubes. Yet, most heat is generated from the "hot spot" and then quickly propagates along the surface of gold nanoparticles and then to carbon nanotubes. ${ }^{[29]}$ This points out that we are measuring an average temperature at the surface of gold nanoparticles. We also assume that the high surface area of carbon nanotubes due to their network distribution in the microparticle shell and higher thermal conductivity of SNWCNT can lead to an increase of heat dissipation upon laser irradiation. This we observed by irradiation of polystyrene microparticles with the same shell composition with the same laser light and power. The softening of the polystyrene microparticles surrounded by the gold nanoshell was observed at a laser power density $9.6 \cdot 10^{4} \mathrm{~W} \cdot \mathrm{cm}^{-2}(\lambda=$ $532 \mathrm{~nm}$ ) which corresponds to $462 \mathrm{~K}$ (Figure 6). Partial melting was also accomplished for this type of particles at identical laser power at shorter exposure time (Figure 6d). The polystyrene cores carrying only SWCNT or SWCNT with less content of gold nanoparticles do not exhibit softening of the polystyrene due to the laser flux. The temperature at which polystyrene was softened is lower than its melting point $\left(\sim 240^{\circ} \mathrm{C}\right)$, however it is above the glass transition temperature $\left(\sim 100^{\circ} \mathrm{C}\right) \cdot{ }^{[30]}$

In order to verify the temperature accuracy derived from Raman spectra, heating experiments in a heating stage were performed. A Linkam THMS600 heating stage is used to study the temperature influence on the Raman spectra of single-walled carbon nanotubes. These results are presented in Figure 7. Figure $7 \mathrm{a}$ shows the temperature derived from Stokes and Anti-Stokes lines induced by electric heating in a Linkam THMS600 stage. The temperatures derived by Equation (1) from the Raman spectra are mainly correlated with those obtained with the heating stage (Figure 7a). The resolution of the thermal measurements was found to be of 5-40 K for laser heating of microparticles with a plasmonic nanostructured shell. The large error bars can be explained by the difference in heating of individual particles. The downshift of the G-band 

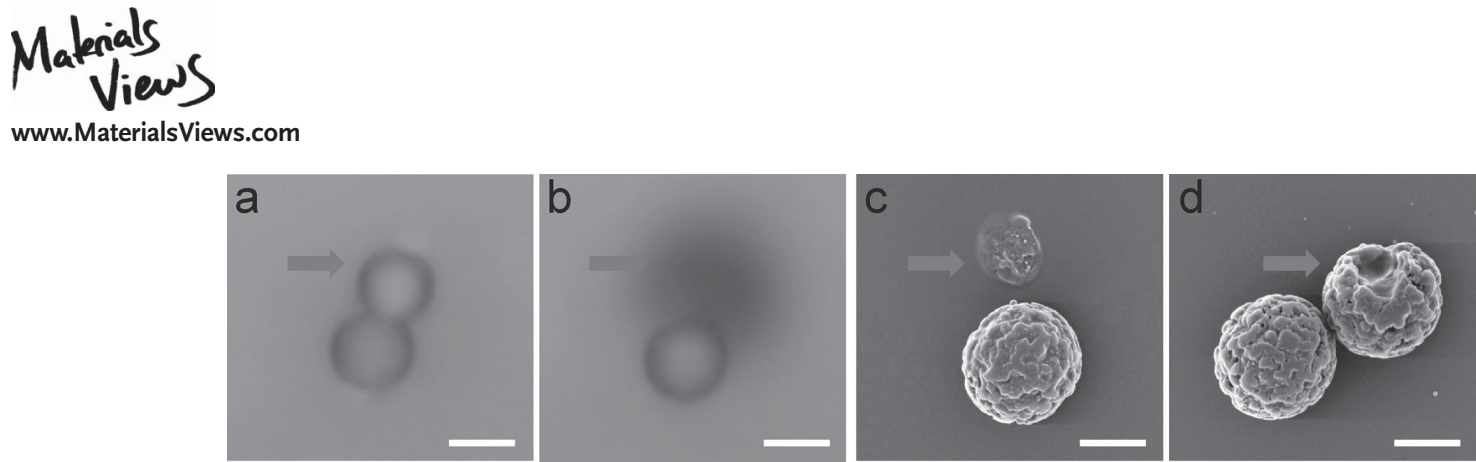

Figure 6. Optical images of polystyrene cores with the shell composition corresponding to PAS composition (a) and these microparticles after laser illumination at a power density of $9.6 \cdot 10^{4} \mathrm{~W} \cdot \mathrm{cm}^{-2}$ (b). SEM images of complete softening of the particle irradiated in b (c), partial melting of the irradiated particle (d). The arrows indicate the irradiated particles. The scale bars correspond to $3 \mu \mathrm{m}$ and $2 \mu \mathrm{m}$ for optical and SEM images respectively.

by laser heating is compatible to that measured by a heating stage (Figure 7b). These changes of frequency of the G-band are pointing out that laser light induces efficient heating of microparticles with a plasmonic nanostructured shell.

\section{Conclusion}

The effective temperature of gold nanoparticle decorated single-walled carbon nanotubes can be derived through acquiring Anti-Stokes and Stokes Raman spectra. Surface plasmons of gold nanoparticles excited with both green and near IR lasers enhance the G-band of single-walled carbon nanotubes. At certain laser energies gold nanoparticles dissipate the heat into carbon nanotubes shifting the G-band. The derived effective temperatures from measurements of Stokes and Anti-Stokes intensities agree with those obtained by electrical heating. The thermal accuracy obtained in this study is $5-40 \mathrm{~K}$. The thermal energy released from gold nanoparticles increases the temperature above the glass transition temperature of polystyrene microparticles enabling softening upon laser illumination. The temperature rise remains stable with time indicating the stability of the adsorption. We envision that our work will help addressing some of the fundamental issues, e.g. spectral fluctuation (SERS blinking effect), dynamic processes in molecular systems as well as applied aspects like temperature assessment from micro- and nanostructured systems, biological entities and photothermal therapy.
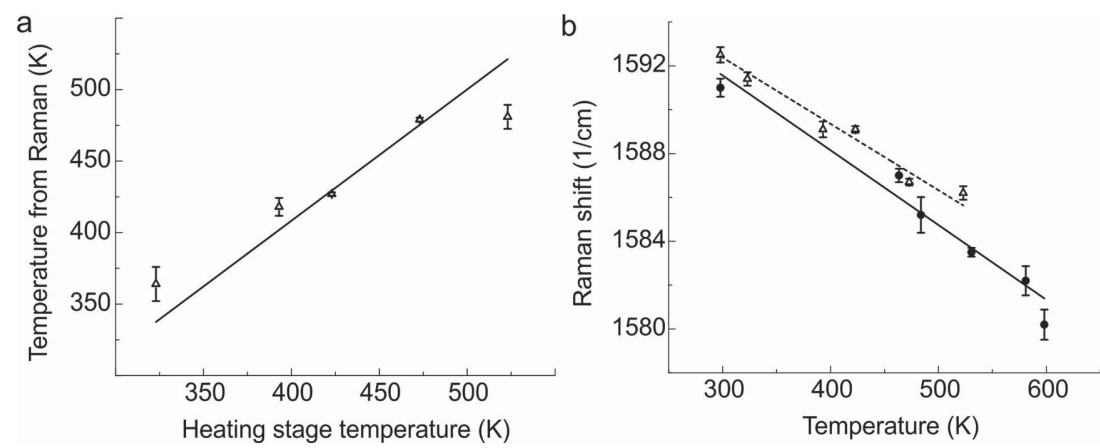

Figure 7. (a) Temperatures calculated from Stokes and Anti-Stokes intensities of Raman spectra obtained by electrical heating (open triangles). (b) Temperature dependence of the frequencies of the G-band for a microprobe covered with single-walled carbon nanotubes subsequently decorated gold nanoparticles by electroless plating heated by a $785 \mathrm{~nm}$ laser (filled circles); and by a Linkam THMS600 stage (open triangles).

\section{Experimental Section}

Materials: A water suspension of silica microparticles $\left(\mathrm{SiO}_{2}\right)$ with average core diameter $4.48 \pm 0.15 \mu \mathrm{m}$, polystyrene cores (PS) having an average diameter $3.63 \pm 0.16 \mu \mathrm{m}$ was purchased from Microparticles GmbH. Poly(sodium 4-styrenesulfonate) (PSS, $70 \mathrm{kDa}$ ) and poly(diallyldimethylammoniumchloride) (PDADMAC, 250-350 kDa), were purchased from Sigma-Aldrich. Sodium chloride $(\mathrm{NaCl})$ and Potassium carbonate $\left(\mathrm{K}_{2} \mathrm{CO}_{3}\right)$ were purchased from Merck. L-ascorbic acid was purchased from Fluka. Positively charged gold nanoparticles (AuNPs) stabilized with 4-(dimethylamino)pyridine (DMAP) were prepared according to previously published methods. ${ }^{[31]}$ Purified HiPco single-walled carbon nanotubes (SWCNT) were purchased from Carbon Nanotechnologies Inc. The surface of carbon nanotubes was functionalized by PSS molecules $(1000 \mathrm{kDa})$ according to previously published methods. ${ }^{[32]}$ In all experiments ultra pure water with resistivity higher than $18.2 \mathrm{M} \Omega$ $\mathrm{cm}$ was used.

LbL Functionalization of Silica Microparticles: First silica and polystyrene microparticles were washed in pure water several times in order to remove artifacts from their surface and to facilitate polyelectrolyte deposition. After washing, polyelectrolytes, carbon nanotubes and gold nanoparticles were adsorbed via Layerby-Layer assembly on the surface of silica and polystyrene beads. $1.8 \mathrm{~mL}$ of water solution of PDADMAC $(2 \mathrm{mg} / \mathrm{mL}$ in $0.5 \mathrm{M} \mathrm{NaCl})$ was added to a silica and polystyrene particle suspension and shaken for $15 \mathrm{~min}$ followed by three washing steps in deionized water. Then the same volume of PSS $(2 \mathrm{mg} / \mathrm{mL}$ in $0.5 \mathrm{M} \mathrm{NaCl})$ was added to the particle suspension, incubated for $15 \mathrm{~min}$ and later washed with water. The deposition of polyelectrolytes was repeated several times resulting in a composition of (PDADMAC/PSS) 3 /PDADMAC. As single-walled carbon nanotubes were stabilized by PSS, they had a negative surface charge (zeta-potential was measured to be $-64.1 \pm 0.2 \mathrm{mV}$ ). The suspension of SWCNT was diluted in pure water with a ratio $1 / 10$ $(\mathrm{v} / \mathrm{v})$ and $1 \mathrm{~mL}$ was injected into the polyelectrolyte modified silica and polystyrene bead suspension. Then the mixture was continuously shaken for 15 min followed by washing in water. The final step includes deposition of gold nanoparticles. Positively charged gold nanoparticles were directly adsorbed on the surface of the SWCNT. The final composition of the nanocomposite multilayered shell on 
the surface of silica and polystyrene microparticles is as follows: (PDADMAC/PSS) ${ }_{3} /$ PDADMAC/SWCNT/AuNPs. A composition of (PDADMAC/PSS) ${ }_{3}$ /PDADMAC/PSS/AuNPs) was analogously constructed around the silica and polystyrene microparticles.

Chemical Growth Procedure: In order to increase the concentration of gold particles on the surface of carbon nanotubes electroless plating via reduction of gold salt was employed. According to the procedure described by T. Pham, the following solution was prepared: $25 \mathrm{mg}$ of $\mathrm{K}_{2} \mathrm{CO}_{3}$ in $100 \mathrm{~mL}$ of deionized water was dissolved and stirred for $10 \mathrm{~min} .{ }^{[33]}$ Then $1.5 \mathrm{~mL}$ of a solution of $1 \%$ $\mathrm{HAuCl}_{4}$ was added and next stirred for $30 \mathrm{~min}$. After that $0.2 \mathrm{~mL}$ of modified silica microparticles were injected to a vigorously stirred $4 \mathrm{~mL}$ growth mediating solution, and $10 \mu \mathrm{L}$ of a solution of $10 \%$ L-ascorbic acid was added. Over the course of $30 \mathrm{sec}$, the solution changed from colorless to pink/blue, which is characteristic of the gold growth. After 2 min of the reaction the silica beads were thoroughly washed with water.

For the further experiments the obtained microparticles were classified as follow:

PST - (PDADMAC/PSS) ${ }_{3} /$ PDADMAC/SWCNT;

PSTA - (PDADMAC/PSS) ${ }_{3} /$ PDADMAC/SWCNT/AUNPS;

PSTAS - (PDADMAC/PSS $)_{3} /$ PDADMAC/SWCNT/AuNPs after electroless plating;

PA - (PDADMAC/PSS) ${ }_{3} /$ PDADMAC/PSS/AuNPs);

PAS - (PDADMAC/PSS) 3 /PDADMAC/PSS/AuNPs) after electroless plating.

Characterization: Scanning electron microscopy (SEM) images were recorded by means of a Philips XL30 electron microscope at an accelerating voltage of $3 \mathrm{kV}$. Field emission Environmental Microscopy (ESEM) was performed with a high-resolution low vacuum FEl Quanda 600 FEG instrument at an operating voltage $30 \mathrm{kV}$ with extended low-vacuum capabilities. Transmission electron microscopy (TEM) images were obtained on a ZeissEM912 Omega transmission electron microscope at an operating voltage of $300 \mathrm{kV}$. Two Raman confocal microscopes operating at two different laser frequencies were used. The first system is a confocal Raman microscope (CRM200, WITec, Ulm, Germany) equipped with a piezo-scanner (P-500, Physik Instrumente, Karlsruhe, Germany) and a diode-pumped $785 \mathrm{~nm}$ NIR laser excitation (Toptica Photonics AG, Graefelfing, Germany). The laser beam was focused through $100 \times$ oil immersion (Nikon, $N A=1.25)$ microscope objective. The spectra were acquired with a thermoelectrically cooled CCD detector (DU401ABV, Andor, UK) behind a grating $\left(300 \mathrm{~g} \mathrm{~mm}^{-1}\right)$ spectrograph (Acton, Princeton Instruments Inc., Trenton, NJ, USA) with a spectral resolution of $6 \mathrm{~cm}^{-1}$. The second system is a confocal Raman microscope (alpha300, WITec, Ulm, Germany) equipped with a frequency doubled Nd:YAG laser (532 nm) laser excitation and piezoscanner (P-500, Physik Instrumente, Karlsruhe, Germany). The spectra were acquired with a thermoelectrically cooled CCD detector (DU401A-BV, Andor, UK) placed behind the spectrometer (UHTS 300; WITec, Ulm, Germany) with a spectral resolution of $3 \mathrm{~cm}^{-1}$. The ScanCtrlSpectroscopyPlus software (version 1.38, Witec) was used for measurement and WITec Project Plus (version 2.02, Witec) for spectra processing. The laser power was measured by a power meter (Newport - Optical Power Meter 1830-C) before the microscope objective. Laser power ranging from 0.5 to $25 \mathrm{~mW}$ at the sample was focused into a spot size of 1-2 $\mu \mathrm{m}$ in diameter (100x objective NA 0.9), giving a power density of $1.6-79.6 \cdot 10^{4} \mathrm{~W} \cdot \mathrm{cm}^{-2}$ at the sample.

\section{Supporting Information}

Supporting Information is available from the Wiley Online Library or from the author.

\section{Acknowledgements}

A. Yashchenok thanks the Alexander von Humboldt-Stiftung for a scholarship. The authors thank to Rona Pitschke and Bat-El Pinchasik for SEM measurements. Financial support was provided by the Max Planck Society and by RFBR project no. 12-03-33088 mol_a_ved and the government of the Russian Federation (grant 14.Z50.31.0004 to support scientific research projects implemented under the supervision of leading scientists at Russian institutions and Russian institutions of higher education). A. Skirtach acknowledges support of FWO (Fonds Wetenschappelijk Onderzoek) Vlaanderen and BOF (Bijzonder Onderzoeksfonds) of Ghent University.

[1] a) A. O. Govorov, H. H. Richardson, Nano Today 2007, 2, 30; b) A. Sanchot, G. Baffou, R. Marty, A. Arbouet, R. Quidant, C. Girard, E. Dujardin, ACS Nano 2012, 6, 3434; c) M. Honda, Y. Saito, N. I. Smith, K. Fujita, S. Kawata, Optics Express 2011, 19, 12375.

[2] a) V. P. Zharov, D. O. Lapotko, IEEE J. Sel. Top. Quant. Electron. 2005, 11, 733; b) D. P. O’Neal, L. R. Hirsch, N. J. Halas, J. D. Paynea, J. L. West, Cancer Lett. 2004, 209, 171; c) H. Shen, J. You, G. Zhang, A. Ziemys, Q. Li, L. Bai, X. Deng, D. R. Erm, X. Liu, C. Li, M. Ferrari, Adv. Healthcare Mater. 2012, 1, 84.

[3] a) A. G. Skirtach, A. A. Antipov, D. G. Shchukin, G. B. Sukhorukov, Langmuir 2004, 20, 6988; b) A. G. Skirtach, P. Karageorgiev, M. F. Bedard, G. B. Sukhorukov, H. Moehwald, J. Am. Chem. Soc. 2008, 130, 11572; c) M. Delcea, N. Sternberg, A. M. Yashchenok, R. Georgieva, H. Bäumler, H. Möhwald, A. G. Skirtach, ACS Nano 2012, 6, 4169.

[4] D. Boyer, P. Tamarat, A. Maali, B. Lounis, M. Orrit, Science 2002, 297, 1160.

[5] a) J. Perez-Juste, B. Rodriguez-Gonzalez, P. Mulvaney, L. M. Liz-Marzan, Adv. Funct. Mater. 2005, 15, 1065; b) 0. Wilson, G. J. Wilson, P. Mulvaney, Adv. Mater. 2002, 14, 1000.

[6] M. Zhu, G. Baffou, N. Meyerbröker, J. Polleux, ACS Nano 2012, 6, 7227.

[7] a) A. S. Urban, T. Pfeiffer, M. Fedoruk, A. A. Lutich, J. Feldmann, ACS Nano 2011, 5, 3585; b) T. Andersen, A. Kyrsting, P. M. Bendix, Soft Matter 2014, DOI: 10.1039/C4SM00410H.

[8] a) J. S. Donner, G. Baffou, D. McCloskey, R. Quidant, ACS Nano 2011, 5, 5457; b) T. Kang, S. Hong, Y. Choi, L. P. Lee, Small 2010, 6, 2649.

[9] D. Jaque, F. Vetrone, Nanoscale 2012, 4, 4301.

[10] a) J. Christofferson, A. Shakouri, Rev. Sci. Instrum. 2005, 76, 024903; b) S. Sadat, A. Tan, Y. J. Chua, P. Reddy, Nano Lett. 2010 , 10, 2613; c) D. Braun, A. Libchaber, Phys. Rev. Lett. 2002, 89, 188103; d) S. Li, K. Zhang, J. M. Yang, L. Liwei, H. Yang, Nano Lett. 2007, 7, 3102; e) G. Baffou, C. Girard, R. Quidant, Phys. Rev. Lett. 2010, 104, 136805 . 
[11] A. G. Skirtach, C. Dejugnat, D. Braun, A. S. Susha, A. L. Rogach, W. J. Parak, H. Möhwald, G. B. Sukhorukov, Nano Lett. 2005, 5, 1371.

[12] A. Takami, H. Kurita, S. Koda, J. Phys. Chem. B 1999, 103, 1226.

[13] M. L. Debasu, D. Ananias, I. Pastoriza-Santos, L. M. Liz-Marzán, J. Rocha, L. D. Carlos, Adv. Mater. 2013, 25, 4868.

[14] a) M. T. Carlson, A. J. Green, H. H. Richardson, Nano Lett. 2012, 12, 1534; b) M. T. Carlson, A. J. Green, A. Khan, H. H. Richardson, J. Phys. Chem. C 2012, 116, 8799.

[15] U. Rocha, C. Jacinto da Silva, W. Ferreira Silva, I. Guedes, A. Benayas, L. Martinez Maestro, M. Acosta Elias, E. Bovero, F. C. J. M. van Veggel, J. A. Garcia Sole, D. Jaque, ACSNano 2013, 7, 1188.

[16] a) T. Beechem, S. Graham, S. P. Kearney, L. M. Phinney, J. R. Serrano, Rev. Sci. Instrum. 2007, 78, 061301; b) M. Kuball, J. M. Hayes, M. J. Uren, T. Martin, J. C. H. Birbeck, R. S. Balmer, B. T. Hughes, IEEE Electron Device Lett. 2002, 23, 7.

[17] H. D. Li, K. T. Yue, Z. L. Lian, Y. Zhan, L. X. Zhou, S. L. Zhang, Z. J. Shi, Z. N. Gu, B. B. Liu, R. S. Yang, H. B. Yang, G. T. Zou, Y. Zhang, S. lijima, Appl. Phys. Lett. 2009, 76, 2053.

[18] D.-H. Chae, B. Krauss, K. von Klitzing, J. H. Smet, Nano Lett. 2010, 10, 466.

[19] S. D. M. Brown, P. Corio, A. Marucci, M. A. Pimenta, K. Kneipp, M. S. Dresselhaus, Phys. Rev. B 2000, 61, R5137.

[20] P. V. Huong, R. Cavagnat, P. M. Ajayan, O. Stephan, Phys. Rev. B 1995, 15, 10048.

[21] J. Kneipp, H. Kneipp, K. Kneipp, Chem. Soc. Rev. 2008, 37, 1052.

[22] a) A. Yashchenok, A. Masic, D. Gorin, B. S. Shim, N. A. Kotov, P. Fratzl, H. Möhwald, A. Skirtach, Small 2013, 9, 351.

[23] S. Hashimoto, D. Werner, T. Uwada, J. Photochem. Photobiol. C 2012, 13, 28.
[24] S. Link, M. A. El-Sayed, J. Phys. Chem. B 1999, 103, 4212.

[25] Y. Niidome, A. Hori, T. Sata, S. Yamada, Chem. Lett. 2000, $29,310$.

[26] D. Werner, S. Hashimoto, J. Phys. Chem. C 2011, 115, 5063.

[27] a) A. G. Skirtach, C. Dejugnat, D. Braun, A. S. Susha, A. L. Rogach, W. J. Parak, H. Möhwald, G. B. Sukhorukov, Nano Lett. 2005, 5, 1371; b) A. G. Skirtach, A. A. Antipov, D. G. Shchukin, G. B. Sukhorukov, Langmuir 2004, 20, 6988.

[28] a) S. Nagasaki, Metal Handbook, 2nd ed., The Japan Institute of Metals, Maruzen, Tokyo, 1984; b) S. P. Hepplestone, A. M. Ciavarella, C. Janke, G. P. Srivastava, Surf. Sci. 2006, 600, 3633; c) J. Che, T. A. Cagin, W. Goddard, Nanotechnology 2000, 11,65 .

[29] a) T. Kang, S. Hong, Y. Choi, L. P. Lee, Small 2010, 6, 2649; b) Y. Seol, A. E. Carpenter, T. T. Perkins, Opt. Lett. 2006, 31, 2429; c) A. Sanchot, G. Baffou, R. Marty, A. Arbouet, R. Quidant, C. Girard, E. Dujardint, ACS Nano 2012, 6, 3434.

[30] J. R. Wunsch, Polystyrene: Synthesis, Production and Applications, Ismithers Rapra Publishing, Shrewsbury, GB, 2000.

[31] D. Gittins, F. Caruso, Angew. Chem. Int. Ed. 2001, 40, 3001

[32] A. M. Yashchenok, D. N. Bratashov, D. A. Gorin, M. V. Lomova, A. M. Pavlov, A. V. Sapelkin, B. S. Shim, G. B. Khomutov, N. A. Kotov, G. B. Sukhorukov, H. Möhwald, A. G. Skirtach, Adv. Funct. Mater. 2010, 20, 3136.

[33] T. Pham, J. B. Jackson, N. J. Halas, T. R. Lee, Langmuir 2002, 18, 4915.

Received: June 11, 2014

Revised: September 1, 2014

Published online: November 3, 2014 